\title{
Quelques réflexions autour de la décentralisation comme objet de recherche
}

Jean-Pierre Olivier de Sardan

\section{OpenEdition}

1 Journals

Édition électronique

URL : https://journals.openedition.org/apad/547

DOI : 10.4000/apad.547

ISSN : 1950-6929

Éditeur

LIT Verlag

Édition imprimée

Date de publication : 1 décembre 1998

\section{Référence électronique}

Jean-Pierre Olivier de Sardan, «Quelques réflexions autour de la décentralisation comme objet de recherche », Bulletin de l'APAD [En ligne], 16 | 1998, mis en ligne le 15 novembre 2006, consulté le 21 septembre 2021. URL : http://journals.openedition.org/apad/547; DOI : https://doi.org/10.4000/apad. 547

Ce document a été généré automatiquement le 21 septembre 2021.

Bulletin de I'APAD 


\title{
Quelques réflexions autour de la décentralisation comme objet de recherche
}

\author{
Jean-Pierre Olivier de Sardan
}

Préliminaire : problèmes de langage

1 Parler de la décentralisation fait presque immanquablement survenir quatre termes-pièges : population, Etat, société civile et chefs traditionnels, qui suscitent vite chacun divers malentendus. Essayons donc de clarifier autant que possible ces problèmes de langage.

Population

2 Je cite un intervenant lors de ces journées (mais chacun d'entre nous a déjà prononcé ce type de phrase un jour ou l'autre): "la population a pris les choses en main en organisant une consultation populaire" (pour désigner son candidat aux élections). Un tel énoncé, qui semble désigner un cas particulièrement heureux de mobilisation collective, satisfait pleinement le bailleur de fonds, le responsable d'ONG ou le cadre politique. Pour le socio-anthropologue, il constitue au contraire un problème, et débouche sur une série de questions, qui sont autant de demandes d'investigations complémentaires: qui a pris l'initiative de cette consultation, soutenu par qui ? pourquoi cette proposition a-t-elle été suivie, au nom de quelle légitimité de ses initiateurs? et a-t-elle vraiment été soutenue par tous ? comment a pu se construire un tel consensus? En effet le consensus n'a rien de naturel, dans un village africain pas plus qu'ailleurs. C'est une production sociale complexe, qui ne va jamais de soi. Quant à la "population", ce n'est jamais un acteur collectif. Une population, cela n'existe pas : c'est un assemblage instable d'acteurs plus ou moins agglomérés en groupes et en réseaux particuliers. C'est l'alchimie toujours renouvelée de ces acteurs, groupes et réseaux qui passionne le socio-anthropologue, et le fait aller toujours au-delà du terme "population", bien qu'il s'agisse d'un raccourci commode, parfois inévitable.

Etat 
Il est tout aussi impossible d'échapper à l'usage de ce mot. Et pourtant, dès qu'on veut lui donner une valeur analytique, il doit éclater en diverses composantes. Par Etat en effet on évoque tantôt l'Institution Etat, avec un grand $\mathrm{E}$, avec ses mythes, sa légitimité et son idéologie, tantôt des appareils d'Etat et des structures administratives, tantôt la classe politique qui dirige ces appareils, voire la politique qu'elle met en œuvre, tantôt une bureaucratie, elle même diverse...

Du point de vue de la décentralisation, on peut au moins dégager deux niveaux totalement différents de l'Etat. D'un côté on aura le gouvernement, et les services sous son autorité directe à la capitale : ce sont les décideurs de la décentralisation, ceux qui la conçoivent, par exemple tantôt de façon plutôt active et souple (cas du Mali), tantôt en la limitant le plus possible (cas du Niger)... De l'autre coté, on aura les représentants locaux de l'Etat, eux-mêmes variés, préfets et sous-préfets, services techniques, personnels administratifs déconcentrés : ce sont les exécutants de la décentralisation, parfois victimes de la réforme, qui renâclent à céder une partie de leurs pouvoirs, parfois au contraire "tuteurs" de la réforme, qui en tirent parti... C'est cette seconde acception de l'Etat, celle qui concerne les représentants étatiques sur la scène locale, qui, en ce qui concerne la mise en œuvre de la décentralisation, c'est-à-dire son insertion dans des arènes villageoises ou urbaines, est particulièrement stratégique et doit faire l'objet de plus d'investigations de notre part.

Société civile

5 Ce terme, issu d'une longue tradition de philosophie politique, a connu une seconde jeunesse récemment, dans le champ du développement, du fait de la montée en puissance des ONG, qui s'en réclament abondamment. Il est de ce fait chargé de connotations politiques et idéologiques "positives", puisqu'il évoque généralement des dynamiques "populaires", "endogènes", "d'en bas", face à son contraire déprécié, l'Etat, sa bureaucratie, son despotisme, ses rigidités...

6 Le socio-anthropologue se méfie en général, pour de bonnes raisons scientifiques, des termes trop connotés moralement. De plus, "société civile", cela semble particulièrement flou, dès lors qu'il s'agirait de tout ce qui n'est pas l'Etat; s'agit-il de l'individu, de la famille, du petit commerce, des entreprises privées, des ONG, des sectes religieuses, etc. ? En fait, on s'aperçoit vite que le référent habituel de cette expression, quand elle prend un sens tant soit peu concret, correspond de fait assez exactement au secteur associatif, ce secteur intermédiaire, cet ensemble d'organisations, groupements, associations en tous genres qui ont fleuri depuis une vingtaine d'années en Afrique, dans le domaine de la production, du développement, de la politique ou de la religion. Parfois, dans le contexte actuel de l'ajustement structurel, on y associera les entrepreneurs privés.

7 Mais alors, pourquoi ne pas se contenter de parler de "secteur associatif" (ou d'entreprise privée), ce qui ferait plus l'affaire du socio-anthropologue? Sans doute parce qu'avec l'expression de "société civile" les associations gagnent un "plus" de légitimité envers les bailleurs de fond: au lieu de ne représenter que ce qu'elles sont, c'est-à-dire un "collectif particulier" composé de leurs propres adhérents, elles représentent "la société civile" (dont elles seraient l"'émanation"), c'est-à-dire donc un peu la société toute entière. De ce fait une simple association peut prétendre sans mandat électif parler au nom d'un intérêt général, et exprimer les "besoins" de la population face à un Etat qui les ignore...

Chef traditionnels 
8 L'une des inconnues de la décentralisation est la place qu'elle accordera, formellement ou informellement, aux "chefs traditionnels". Mais qui sont ces fameux "chefs traditionnels" ? Les sociétés pré-coloniales connaissaient différentes formes de pouvoir politique local, qu'on a traduit en français sous des noms divers, depuis les plus minuscules (doyen d'un segment de lignage, aîné d'un hameau) jusqu'aux plus vastes, parfois plus ou moins "fédératives" (émirats, royaumes, confédérations), en passant par les plus habituelles, regroupant un bourg ou quelques villages sous l'autorité d'un "chef", parfois simple primus inter pares, parfois aristocrate membre d'une "maison" régnante (chefferies). Mais ces formes ont été partout remplacées sous la domination française par l'institution de la chefferie administrative (chefs de village, de canton, ou chefs supérieurs), dernier échelon de la chaîne de commandement coloniale. Ces chefferies administratives ont cependant tenté, avec plus ou moins de succès, de se donner une légitimité "traditionnelle", et de se présenter comme le prolongement "naturel" des formes politiques anciennes. Dans les Etats post-coloniaux, la chefferie administrative est parfois restée jusqu'à nos jours la forme de base du pouvoir local (en particulier au Niger, avec l'instance stratégique de la chefferie de canton, non remise en cause dans l'actuelle décentralisation, mais aussi sous des formes plus atténuées en Côte d'Ivoire ou au Togo). Ailleurs, la chefferie a été supprimée comme instance officielle, mais les descendants des chefs administratifs, ainsi que les descendants des chefs pré-coloniaux (ce sont parfois les mêmes, mais pas toujours) ont souvent relevé la tête dans la foulée des actuelles réformes de décentralisation et tentent de regagner une influence politique.

9 Ainsi, un "chef traditionnel" ce peut être tantôt un chef administratif officiel encore en fonction, et tantôt un chef sans statut administratif mais se réclamant d'une légitimité soit pré-coloniale, soit coloniale (mais se présentant comme si elle était pré-coloniale.. .). De plus un chef peut avoir aujourd'hui des fonctions sociales fort diverses, parfois foncières, parfois rituelles, parfois "folkloriques", parfois arbitrales...

10 En tout cas, le contexte actuel de la décentralisation n'a rien de "traditionnel", et ne saurait être confondu avec un retour à des formes pré-coloniales de prises de décision locale. La décentralisation, partout, consiste à mettre en place des autorités communales élues au suffrage universel dotées de ressources et pouvoirs nouveaux.

Le contexte de la décentralisation en Afrique

11 On peut mettre l'accent sur au moins trois tendances majeures, même s'il en est bien sûr d'autres.

Tendance à la reproduction en contexte démocratique d'une partie de la culture politique des régimes autoritaires post-coloniaux

12 Le passage au multipartisme n'a pas constitué une disparition de la culture politique du parti unique, mais plutôt sa démultiplication. C'est ce qu'exprime la boutade qui affirme que, aujourd'hui, où il existe des dizaines de partis, chaque parti se comporte comme s'il était (ou voulait devenir) un parti unique. Le parti au pouvoir tend à éviter par tous les moyens (en particulier en recourant à la fraude massive) l'alternance. Quand celle-ci survient, ce qui reste rare, au moins de façon pacifique, le parti qui l'emporte accapare les postes et les distribue de haut en bas de l'échelle administrative, parfois jusqu'au niveau du planton, sur des bases d'affiliation partidaire et non en fonction de critères de compétence. Quand il y a gouvernement de coalition, le même phénomène intervient selon les chasses gardées de chaque parti. On pourrait parler 
d'une dérive "partidiste". Autre manifestation de cette tendance: la stratégie systématique du "bras de fer" entre majorité et opposition.

De même le mode néo-patrimonialiste de gouvernance, et le prélèvement ou la distribution de prébendes liés à l'occupation de fonctions d'autorité n'ont pas en général été freinés par l'actuelle démocratisation, mais semblent même avoir été parfois amplifiés. Les alternances éventuelles introduisent simplement une rotation des prébendes, qui a pu amener certains à évoquer une sorte de tontine... Plus généralement l'extension de la corruption sous les régimes autoritaires ou dictatoriaux n'a guère connu de coup d'arrêt à ce jour avec les régimes démocratiques.

On comprend de ce fait que les régimes démocratiques qui mettent en oeuvre la décentralisation aujourd'hui se heurtent à une méfiance générale dans le pays, le discrédit des classes politiques étant plus marqué que jamais. Les attitudes populaires face à la décentralisation sont bien évidemment marquées par un tel contexte.

Tendance à une dépendance croissante envers l'extérieur

Que ce soit une dépendance à l'égard des divers "bailleurs" (dépendance envers la "rente du développement"), que ce soit une dépendance à l'égard du FMI et de l'ajustement structurel (dépendance envers les politiques économiques imposées), la marge de manœuvre des pouvoirs en place s'est singulièrement réduite ces dernières années. Or la décentralisation est de plus en plus une priorité des bailleurs, et fait figure de plus en plus de conditionnalité. Si cette incitation externe forte rencontre parfois un accord réel de la part d'un gouvernement (cas du Mali) ${ }^{1}$, beaucoup de régimes africains y consentent plutôt en reculant.

Tendance à l'affaiblissement, au démembrement ou à l'émiettement de nombreuses fonctions étatiques

La faillite largement reconnue des classes politiques, sous les régimes de parti unique, les régimes militaires, et les régimes démocratiques, a conduit à d'innombrables disfonctionnements d'un service public devenu aussi pléthorique qu'inefficace, désormais incapable de remplir certaines de ses fonctions de base. Cette situation, qui a pour une grande part légitimé l'ajustement structurel, n'a guère été modifiée, au contraire, par le-dit ajustement. De plus en plus de fonctions étatiques sont désormais assurées de façon anarchique par ces sortes d'enclaves que constituent les "projets". Un appareil d'Etat à deux vitesses s'est mis ainsi progressivement en place : l'appareil d'Etat central classique, de plus en plus dévalorisé et déliquescent, sans ressources, réglé par un standard national de façade et utilisant des fonctionnaires sous-payés et inefficaces, et des bouts d'appareils d'Etat semi-privés, alimentés et controlés directement par les bailleurs, aux normes internationale, embauchant des cadres nationaux bien payés.

Ce qui reste de l'Etat central semble de plus en plus incapable de produire des règles du jeu acceptables par tous, autrement-dit de proposer des normes de référence qui fassent foi (en matière foncière, comme dans le rapport administration-administrés, ce phénomène est fort visible). La pluralité et l'instabilité des normes, phénomènes déjà observables depuis longtemps, en sont accrues.

18 La profusion de formules juridiques, constitutionnelles, administratives qui se sont succédées depuis un siècle dans chaque pays africain, la valse incessante des réformes, l'instabilité des règles du jeu politique, ajoutent encore à l'incertitude. 
19 Enfin, la déliquescence de l'Etat s'exprime par une recrudescence générale de la criminalité, de l'insécurité, de la violence, voire par des menaces sérieuses sur la paix civile.

On voit que les effets cumulés de ces trois tendances pèsent d'un poids lourd sur les réformes décentralisatrices. Si celles-ci ignorent un tel contexte ou n'en tiennent pas compte, les effets pervers ne risquent-ils pas de se multiplier? On connait en effet les dérives de tous ordres (clientélistes, mafieuses, régionalistes, ethnicistes, intégristes) qu'une décentralisation peut susciter.

Quatre questions centrales pour une socio-anthropologie de la decentralisation

Il en est certes d'autres, plus concrètes, et non moins importantes ${ }^{2}$. Mais ces quatre là nous semblent constituer à la fois des enjeux politiques et des enjeux scientifiques majeurs.

La question des modes locaux de gouvernance

On a pu parler aussi des régimes politiques locaux, des gouvernementalités locales, peu importe. Partout il existe des formes, rurales ou urbaines, de gouvernance de proximité, souvent relevant d'une combinaison de centres de pouvoir multiples, en partie absents des organigrammes et des textes juridiques. Or la décentralisation devrait, si elle est mise en œuvre de façon sérieuse (ce qui n'est pas acquis partout), constituer un changement important. Elle devrait avoir des effets structurants forts sur les modes locaux de gouvernance. Une série d'interrogations en découle. Quelles fonctions étatiques seront assurées par les nouvelles collectivités locales, et comment ? Pourront-elles mieux contrôler la violence et arbitrer les conflits ? Seront-elles à même de produire des normes et régulations locales légitimes et reconnues dans la pratique par la majorité des acteurs? Les classes politiques locales reproduiront-elles au niveau des villages ou des quartiers, grâce à la décentralisation, la culture politique nationale, en la diffusant "en bas" (partidisme, néo-patrimonialisme, corruption, etc.), ou s'en démarqueront-elles? Au Sénégal, la décentralisation semble avoir permis aux partis et surtout aux factions du PS de s'implanter localement et de procurer aux cadres locaux des enjeux et des ressources à leur mesure : est-ce le processus qui sera suivi ailleurs ? Assistera-t-on au développement d'une bureaucratie locale, quels nouveaux rapports s'établiront avec les agents déconcentrés de l'Etat?

La question de l'éligibilité au pouvoir local

Il s'agit de savoir qui aura accès aux nouvelles instances de pouvoir, et, plus largement, qui est susceptible d'y revendiquer une place. Qui est promouvable aux conseils communaux, aux fonctions de maire ? Y aura-t-il main mise sur les nouvelles fonctions des notabilités locales et titulaires de charges plus ou moins officieuses, ou promotion d'une nouvelle élite locale, issue par exemple du mouvement associatif ou syndical ? Un des objectifs déclarés ou non dits de la décentralisation est l'empowerment: qu'en serat-il réellement? N'assiste-t-on pas parfois à une recrudescence, au contraire, de phénomènes d'exclusion? Parfois, ces deux tendances contradictoires peuvent aller de pair : rien n'empêche que se produise simultanément une exclusion des allochtones et une promotion des jeunes natifs, par exemple...

C'est aussi tout le problème du rapport entre éligibilité et clientélisme qui est posé. Trois cas de figures peuvent se présenter: l'usage, dans le cadre des nouvelles collectivités locales, de réseaux clientélistes déjà en place localement ; le remplacement d'un réseau clientéliste existant par un autre, plus ou moins émergent : l'implantation à 
la faveur de la décentralisation d'un nouveau réseau clientéliste là où il n'en existait pas...

La question de la communalité

25 Les interrogations portent ici sur les modalités éventuelles de constitution d'un espace public local, d'un bien public local, d'un service public local, d'un civisme local. Bien souvent, les arènes de villages, de quartiers ou de villes sont caractérisées par la coexistence ou l'affrontement de collectifs particuliers, plus ou moins hiérarchisés, et n'offrent pas de tradition de type "communal". Les processus de constitution d'un espace public commun sont à n'en pas douter complexes et longs. Comment, avec la décentralisation, vont se négocier les rapports entre les nouvelles collectivités locales et les associations existantes ? Le "modèle communal" ne peut hâtivement être assimilé au "modèle associatif". L'un va-t-il se superposer à l'autre, vont-ils entrer en concurrence') Souvent les ONG et "projets" conçoivent la constitution de groupement et autres "comités" comme une pédagogie civique et une étape vers l'auto-gestion de collectivités locales: la route est-elle aussi droite, les comités ne sont-ils pas des collectifs particuliers plus que les représentants d'un interêt général? Les conseils municipaux vont-ils à leur tour se comporter en collectifs particuliers, ou bien développer petit à petit des normes pratiques relevant de l'interêt général ? Les efforts des collectivités locales pour mobiliser sur le plan fiscal des ressources locales enclencheront-ils ces mêmes mécanismes de soupçon (de "détournement") qui pèsent généralement sur les bureaux des associations? Y aura-t-il au fond production progressive d'une légitimité municipale?

La question des usages stratégiques de la décentralisation

De façon presque inverse, la décentralisation peut aussi être appréhendée à partir d'une perspective "stratégique", autrement dit comme un enjeu autour duquel des acteurs ou des groupes d'acteurs s'affairent en vue d'en tirer des avantages particuliers. Toute redistribution, même partielle des cartes, s'opère au sein d'un jeu déjà en cours. Toute introduction de pouvoirs nouveaux ou de ressources nouvelles se greffe sur un fond d'intrigues déjà en place. Le problème, avec une telle perspective, serait de décliner à l'infini les raffinements ou les variations stratégiques des uns et des autres et de s'en satisfaire, ou de n'en sortir qu'au profit de typologies purement formelles, ou formalistes. Les usages stratégiques de ressources nouvelles telles que la décentralisation doivent au contraire être référés à des enjeux précis, à des ressources précises, et à des contraintes précises. La référence aux contextes et aux rapports de force s'impose.

Prenons un exemple, les réseaux sociaux, qui étaient un des thèmes centraux de ces Journées, un peu oublié. Il n'est guère productif de s'en tenir au constat que chaque acteur a ses réseaux sociaux propres, et qu'il en joue à sa façon. Mieux vaut considérer que les réseaux eux-mêmes peuvent être à la fois des enjeux, des ressources, et des contraintes. Les réseaux peuvent être des enjeux de la décentralisation, dans la mesure où l'accès à des responsabilités municipales peut permettre de s'insérer dans de nouveaux réseaux, ceux, internationaux, de la coopération décentralisée et des jumelages, par exemple, ou ceux, nationaux, des élites régionales, voire nationales. Les réseaux sont aussi à l'évidence des ressources, qui favorisent l'éligibilité aux fonctions décentralisées, que ce soit l'appartenance à des réseaux confessionnels ou partidaires, ou la capacité d'attirer des "projets" via des ONG du Nord. Enfin, les réseaux sont aussi des contraintes, car les responsables de collectivités locales vont se trouver obligés de 
redistribuer certains "bénéfices" de leurs fonctions à leurs réseaux d'appartenance, familiaux ou clientélistes par exemple.

Problèmes de méthode

Manifestement, la recherche sur les processus de décentralisation, comme ces processus eux-mêmes, ne fait que commencer, et doit s'approfondir. Dans une perspective comparative, c'est sans doute la question de la mise au point d'indicateurs qui est cruciale. Or il est des questions pour lesquelles on voit mal comment construire des indicateurs. Prenons certains des objectifs explicites majeurs de la décentralisation, le civisme, la démocratie, la participation, voire d'autres adjacents comme la confiance ou le consensus : comment évaluer les effets de la décentralisation sur ces variables de façon fiable, c'est-à-dire autrement qu'impressionniste, ou en évitant les jugements de valeur?

Heureusement, il est d'autres domaines, où il semble plus envisageable de construire des indicateurs (quantitatifs, mais aussi qualitatifs) à partir de questions de recherche telles que ${ }^{3}$

- la capacité de la décentralisation à améliorer les infrastructures locales et surtout leur maintenance, comme à proposer des services ou à améliorer la qualité des services de type "étatique" existant :

- les effets de la décentralisation sur les structures associatives :

- en quoi la décentralisation augmente-t-elle ou diminue-t-elle la corruption, et les soupçons ?

- la décentralisation permet-elle un renouvellement des élites politiques locales?

- la décentralisation a-t-elle un effet sur la violence?

- les partis politiques tirent-ils parti localement de la décentralisation?

- la décentralisation permet-elle une meilleure coordination des "projets", voire un contrôle de ceux-ci lorsqu'ils interfèrent avec l'espace public?

Il est bien sûr nombre d'autres questions de recherche pertinentes, et susceptibles de permettre une construction d'indicateurs... au moins indicatifs.

Il reviendra donc à tous ceux qui mènent des travaux empiriques sur la décentralisation de confronter leurs expériences et leurs acquis en la matière. Ce chantier n'en est clairement qu'àses débuts.

\section{NOTES}

1.Rappelons ici que le Sénégal a commencé à anticiper de son propre chef dès 1972 les actuelles réformes.

2.A. S. Fall en a ainsi signalé quelques-unes lors du débat : la translocalisation croissante et les phénomènes migratoires ; la question des ressources des collectivités locales et la fiscalité ; les capacités des élus locaux à gérer les ressources naturelles sur le long terme et l'aménagement du territoire...

3.Le passage d'une question de recherche à un indicateur est évidemment un processus de construction qui ne peut se faire qu'en référence à des enquêtes empiriques. Nous en 
resterons donc ici aux seules questions de recherche pour lesquelles un tel processus semble possible.

\section{AUTEUR}

JEAN-PIERRE OLIVIER DE SARDAN

EHESS-CNRS, 2 rue de la Vieille Charité, F-13002 Marseille (France). Tél. +33 (0)4 91 140772 Fax. +33 (0)4 91913401 Email : jpos@ehess.cnrs-mrs.fr 